\title{
THE MODULE STRUCTURE OF SOLOMON'S DESCENT ALGEBRA
}

\section{DIETER BLESSENOHL and HARTMUT LAUE}

(Received 14 October 1997; revised 24 April 2001)

Communicated by J. Ramagge

\begin{abstract}
A close connection is uncovered between the lower central series of the free associative algebra of countable rank and the descending Loewy series of the direct sum of all Solomon descent algebras $\Delta_{n}$, $n \in \mathbb{N}_{0}$. Each irreducible $\Delta_{n}$-module is shown to occur in at most one Loewy section of any principal indecomposable $\Delta_{n}$-module. A precise condition for this occurrence and formulae for the Cartan numbers are obtained.
\end{abstract}

2000 Mathematics subject classification: primary 16N20; secondary 16D15, 16G10, 20C30, $05 \mathrm{E} 15$. Keywords and phrases: descent algebra, principal indecomposable modules, Loewy series, lower central series, Poincaré-Birkhoff-Witt bases, Cartan numbers, free associative algebra.

\section{Introduction}

In [12], Solomon introduced a remarkable family of algebras associated with Coxeter groups. In the special case of symmetric groups, these are called descent algebras and have been studied since then by various authors. A surprising number of intricate and fascinating results have been obtained during the last decade (see, for example, $[1,6],[7$, Section 5], [11, Chapter 9]). For any positive integer $n$, the descent algebra $\Delta_{n}$ is a subalgebra of the group ring $K S_{n}$ and may be described as the $K$-linear span of the elements $\delta_{D}:=\sum_{\sigma \in S_{n}(D)} \sigma, D \subseteq\{1, \ldots, n-1\}$, where $S_{n}(D)$ is the set of all permutations with descent set $D$. (Recall that the descent set of $\sigma \in S_{n}$ consists of all $j<n$ such that $j \sigma>(j+1) \sigma$.) We set $\Delta_{0}:=K$ and $\Delta:=\bigoplus_{n \geq 0} \Delta_{n}$, the direct $K$-algebra sum of all $\Delta_{n}$. The ground ring $K$ will throughout be assumed to be a field of characteristic 0 .

(C) 2002 Australian Mathematical Society $1446-7887 / 2000 \$ A 2.00+0.00$ 
For every associative $K$-algebra $A$ we put $\gamma^{(0)}(A):=A$ and define inductively $\gamma^{(j+1)}(A)$ to be the ideal generated by all elements $x y-y x\left(x \in \gamma^{(j)}(A), y \in A\right)$. Then $\left(\gamma^{(j)}(A)\right)_{j \geq 0}$ is called the lower central series of $A$.

In this paper we give a $K$-linear isomorphism $\omega$ of the free associative algebra $F$ of countable rank onto $\Delta$ such that

(i) $\gamma^{(j)}(F)$ is mapped onto $(\operatorname{Rad} \Delta)^{j}$ for all $j \geq 0$;

(ii) the multihomogeneous components of $F$ are mapped onto a direct decomposition of $\Delta$ into indecomposable left ideals.

Our first main result (Theorem 2.1) may be interpreted as follows:

(j) $(\operatorname{Rad} \Delta)^{j}$ is the $j$ th term of the lower central series of $(\Delta,+, *)$,

where $*$ is the convolution product in the sense of Reutenauer $[11,(1.5 .3), 9$.$] . This is$ due to the fact that $\omega$ is an algebra isomorphism of $F$ onto $(\Delta,+, *)$, as was pointed out to us by Jöllenbeck.

In our second main result (Theorem 2.2), we describe the occurrence of the irreducible $\Delta_{n}$-modules in the Loewy sections of any principal indecomposable $\Delta_{n}$-module. Furthermore, if $\mathfrak{B}$ is any Poincaré-Birkhoff-Witt basis of $F$, then $\mathfrak{B} \omega \cap(\operatorname{Rad} \Delta)^{j}$ is a basis of $(\operatorname{Rad} \Delta)^{j}$, for every non-negative integer $j$. In particular, we obtain a complete description of the Cartan matrix of $\Delta_{n}$ (Corollary 2.1).

The results of [4], in particular [4, 1.5, 2.5], are of crucial importance for this paper.

\section{Principal indecomposable $\Delta_{n}$-modules and irreducible $\Delta_{n}$-modules}

Let $\mathbb{N}^{*}$ be the free monoid over the alphabet $\mathbb{N}=\{1,2,3, \ldots\}$. We put $\mathbb{N}_{0}:=$ $\mathbb{N} \cup\{0\}$ and $m:=\{i \mid i \in \mathbb{N}, i \leq m\}$ for all $m \in \mathbb{Z}$. For every finite set $A$, we write $S_{A}$ for the symmetric group on $A$ and set $S_{m}:=S_{m}$. For every $q, r \in \mathbb{N}^{*}$, we write $q r$ for the concatenation of $q$ and $r$. The $j$ th letter of the word $q \in \mathbb{N}^{*}$ will be denoted by $q_{j}$. If $q=q_{1} \cdots q_{k} \in \mathbb{N}^{*}, k \in \mathbb{N}_{0}$, we set $\operatorname{sum}(q):=\sum_{j \in k} q_{j} \in \mathbb{N}_{0}$. If $\operatorname{sum}(q)=n$, then $q$ is called a composition of $n, k$ its length, for which we use the notation $q \vDash n,|q|=k$ respectively. Putting $Q_{j}:=\left(q_{1}+\cdots+q_{j-1}\right)+q_{j}$ for all $j \in \underline{k}$, we set $\mathscr{S}^{q}:=\left\{\sigma\left|\sigma \in S_{n}, \sigma\right|_{Q_{j}}\right.$ is increasing, for all $\left.j \in \underline{k}\right\}$ and

$$
\mathscr{X}^{q}:=\left\{\sigma\left|\sigma \in S_{n}, Q_{j}^{\sigma}=Q_{j}, \sigma\right|_{Q_{j}} \text { has a unique local minimum, for all } j \in \underline{k}\right\} .
$$

The defect of any permutation $\sigma \in S_{n}$ is defined by

$$
d(\sigma):=|\{j \mid j \in \underline{n-1}, j \sigma>(j+1) \sigma\}| .
$$

Let $\Xi^{q}:=\sum_{\sigma \in \mathscr{S}^{q}} \sigma$ and $\omega_{q}:=\sum_{\sigma \in \mathscr{X}^{q}}(-1)^{d(\sigma)} \sigma \Xi^{q}$. Then $\left\{\Xi^{q} \mid q \vDash n\right\}$ is a $K$-basis of $\Delta_{n}$ ([12]). If $q=q_{1} \cdots q_{k}, r=r_{1} \cdots r_{l} \models n$, then $\Xi^{q}$ and $\Xi^{r}$ are congruent modulo 
the Jacobson radical $\operatorname{Rad} \Delta_{n}$ of $\Delta_{n}$ if and only if $r$ is associate to $q$, that is, if and only if $k=l$ and there exists a permutation $\varrho \in S_{k}$ such that $\varrho q:=q_{1 \varrho} \cdots q_{k_{\ell}}$ coincides with $r$. In this case we write $q \approx r$. The set $\left\{\omega_{q} \mid q \models n\right\}$ is also a $K$-basis of $\Delta_{n}$, and $\Lambda^{q}:=\Delta_{n} \omega_{q}$ is an indecomposable left ideal of $\Delta_{n}$, for every $q \models n([4,1.2,1.3])$. A partition is a word $p \in \mathbb{N}^{*}$ such that $p_{1} \geq p_{2} \geq \cdots$, and if $p$ is a partition such that $\operatorname{sum}(p)=n$, we write $p \vdash n$. For later use, we record the following elementary fact:

The number of all partitions $p$ of length $k$ such that

$$
p_{j} \leq m \text { for all } j \in \underline{k} \text { is equal to }\left(\begin{array}{c}
m+k-1 \\
k
\end{array}\right)
$$

for every $m, k \in \mathbb{N}_{0}$. For every word $q \in \mathbb{N}^{*}$ there exists a unique partition $p$ such that $p \approx q$. We have

$$
\Delta_{n}=\bigoplus_{q \vdash n} \Lambda^{q} \quad \text { for all } n \in \mathbb{N}
$$

If $q=q_{1} \cdots q_{k} \models n$, we set $q$ ? := $\prod_{i \in \underline{k}} q_{i} \prod_{j \in \underline{n}}\left(a_{j}(q) !\right)$ where $a_{j}(q):=\mid\{i \mid i \in$ $\left.\underline{k}, q_{i}=j\right\} \mid$. Then

$$
\nu_{q}:=\frac{1}{q ?} \omega_{q}
$$

is an idempotent generator of the indecomposable left ideal $\Lambda^{q}$ of $\Delta_{n}([4,1.3,(27)])$. Therefore it is a primitive idempotent, and $\left(\operatorname{Rad} \Delta_{n}\right) \nu_{q}=\operatorname{Rad}\left(\Delta_{n} \nu_{q}\right)$ is the unique maximal element of the set of all left ideals of $\Delta_{n}$ which are properly contained in $\Lambda^{q}([5,41.2,45.7])$. In general, we write $\operatorname{Rad} M$ for the intersection of all maximal submodules of a module $M$, and we set $\operatorname{Rad}^{(0)} M:=M, \operatorname{Rad}^{(j)} M:=\operatorname{Rad}\left(\operatorname{Rad}^{(j-1)} M\right)$ for all $j \in \mathbb{N}$. By $[4,1.6], v_{q} \equiv v_{r} \bmod \operatorname{Rad} \Delta_{n}$ if and only if $q \approx r$, for any $q, r \vDash n$. It follows that $\left\{\operatorname{Rad} \Delta_{n}+v_{p} \mid p \vdash n\right\}$ is the set of primitive idempotents of $\Delta_{n} / \operatorname{Rad} \Delta_{n}$. In particular, for all $q, r \models n$

$$
v_{q} \nu_{r} \in \operatorname{Rad} \Delta_{n} \text { if and only if } q \not \approx r .
$$

For all $q \vDash n$, we set $M^{q}:=\left(\operatorname{Rad} \Delta_{n}+\left\langle v_{q}\right\rangle\right) / \operatorname{Rad} \Delta_{n}$. Then $\left\{M^{p} \mid p \vdash n\right\}$ is a complete set of irreducible $\Delta_{n}$-modules. (As $\Delta_{n} / \operatorname{Rad} \Delta_{n}$ is commutative, there is no distinction between irreducible representations and anti-representations of $\Delta_{n}$, that is, between irreducible $\Delta_{n}$-right modules and left modules.) By means of (3), the following proposition is readily verified.

PROPOSITION 1.1. Let $n$ be a positive integer, $p \vdash n$, and let $\eta$ be an idempotent of $\Delta_{n}$ such that $\eta \equiv v_{p} \bmod \operatorname{Rad} \Delta_{n}$. Let $N$ be a finite-dimensional (left or right) $\Delta_{n}$-module and $N^{\prime}$ the image of $N$ under $\eta$. 
(a) A composition factor of $N$ is not annihilated by $\eta$ if and only if it is isomorphic to $M^{p}$.

(b) $\operatorname{dim}_{K} N^{\prime}$ is the multiplicity of $M^{p}$ in a composition series of $N$.

We shall need the following extension of the notion of composition. If $r, s \in \mathbb{N}^{*}$, $k:=|s|$ and there exist elements $r[1], \ldots, r[k] \in \mathbb{N}^{*}$ such that $r=r[1] \cdots r[k]$ and $r[j] \vDash s_{j}$ for all $j \in \underline{k}$, then $r$ is called a composition of $s$, for which we also use the notation $r \vDash s$. Then $r[1], \ldots, r[k]$ are uniquely determined, and we set $r(s)_{j}:=r[j]$ for all $j \in \underline{k}$. (Here we have deliberately altered the notation introduced in $[4,(4)]$, which was pointed out as incorrect to us by careful readers.) Moreover, we write $r \sim s$ if there exists an element $r^{\prime} \in \mathbb{N}^{*}$ such that $r \approx r^{\prime} \vDash s$. Obviously, $\sim$ induces a partial order on the set of all partitions. Writing $\leq_{\text {lex }}$ for the usual lexicographic order of $\mathbb{N}^{*}$, we observe

$$
q \sim p \Longrightarrow q \leq_{\text {lex }} p \quad\left(q \in \mathbb{N}^{*}, p \text { partition }\right)
$$

From $[4,1.5]$ we conclude that

$$
v_{r} v_{q}=0 \text { for all } q, r \vDash n \text { such that } q \not \not<r
$$

(where $q \not \nvdash r$ means, as usual, that $q \sim r$ does not hold). If $\varepsilon_{1}, \ldots, \varepsilon_{m}$ are idempotents of an associative algebra such that $i>j$ implies $\varepsilon_{i} \varepsilon_{j}=0$, then the elements $\varepsilon^{(k)}:=$ $\left(1-\varepsilon_{1}\right) \cdots\left(1-\varepsilon_{k-1}\right) \varepsilon_{k}, k \in \underline{m}$, are a system of mutually orthogonal idempotents, and $\varepsilon_{i}\left(\varepsilon^{(1)}+\cdots+\varepsilon^{(m)}\right)=\varepsilon_{i}=\left(\varepsilon^{(1)}+\cdots+\varepsilon^{(m)}\right) \varepsilon_{i}$ for all $i \in \underline{m}$. These observations yield:

PROPOSITION 1.2. Let $p_{1}, \ldots, p_{m}$ be the partitions of a positive integer $n$, in lexicographically decreasing order. Put $v^{(k)}:=\left(\mathrm{id}-v_{p_{1}}\right) \cdots\left(\mathrm{id}-v_{p_{k-1}}\right) v_{p_{k}}$ for all $k \in \underline{m}$. Then $\left\{v^{(1)}, \ldots, v^{(m)}\right\}$ is a system of mutually orthogonal idempotents, the subalgebra $\left\langle v^{(1)}, \ldots, v^{(m)}\right\rangle_{K}$ is a complement of $\operatorname{Rad} \Delta_{n}$ in $\Delta_{n}$, and $v^{(1)}+\cdots+v^{(m)}=\mathrm{id}$.

For every $q \vDash n$ we set $\mathrm{P}^{q}:=\nu_{q} \Delta_{n}$. Then $\mathrm{P}^{q}$ is a one-headed right ideal of $\Delta_{n}[5$, 41.2, 45.7]. We remark:

$$
\Delta_{n}=\bigoplus_{q-n} \mathrm{P}^{q}
$$

This is a consequence of (2) and the following

Proposition 1.3. Let $K$ be a field and $A$ a finite-dimensional associative $K$ algebra with unity such that $A / \operatorname{Rad} A$ is commutative. Let $e_{1}, \ldots, e_{k}$ be primitive idempotents of $A$. Then

$$
A=\bigoplus_{j=1}^{k} A e_{j} \quad \text { if and only if } \quad A=\bigoplus_{j=1}^{k} e_{j} A
$$


(Easy examples show that the conclusion does not necessarily hold if $A / \operatorname{Rad} A$ is not assumed to be commutative.)

PROOF. It suffices to prove only one implication. From $A=\bigoplus_{j=1}^{k} A e_{j}$ we conclude that $\bar{A}=\bigoplus_{j=1}^{k} \bar{A} \overline{e_{j}}=\bigoplus_{j=1}^{k} \overline{e_{j}} \bar{A}$, where the upper bar denotes the canonical epimorphism of $A$ onto the commutative quotient $A / \operatorname{Rad} A$. We now have $\operatorname{Rad} A+\sum_{j=1}^{k} e_{j} A=A$. As $\sum_{j=1}^{k} e_{j} A$ is a right ideal of $A$, this implies $\sum_{j=1}^{k} e_{j} A=A$. But $e_{j} A$ is a principal indecomposable $A$-module by [5, 41.2], for all $j \in \underline{k}$, and $e_{j} A \supsetneqq e_{i} A$ for $i \neq j$ because $\overline{e_{j} A} ¥ \overline{e_{i} A}$. Hence the sum of the right ideals $e_{j} A$ is a direct sum.

For every $q \vDash n$, we have $v_{q} \in \Lambda^{q} \cap \mathrm{P}^{q}$. (In fact, from $[4,1.5]$ it follows that $\left\langle v_{q}\right\rangle_{K}=\Lambda^{q} \cap \mathrm{P}^{q}$.) Hence the algebra $\left\langle\nu_{q}\right\rangle_{K}$ is a complement of $\operatorname{Rad} \Lambda^{q}$ in $\Lambda^{q}$ and of $\operatorname{Rad} \mathrm{P}^{q}$ in $\mathrm{P}^{q}$. Therefore we have

$$
\Lambda^{q} / \operatorname{Rad} \Lambda^{q} \cong M^{q} \cong \mathrm{P}^{q} / \operatorname{Rad} \mathrm{P}^{q}\left(\text { as } \Delta_{n} \text {-modules }\right) .
$$

Now $[5,45.7]$ implies that, for any $q, r \models n$,

$$
\Lambda^{q} \cong \Lambda^{r} \text { (as } \Delta_{n} \text {-left modules) if and only if } q \approx r
$$$$
\text { if and only if } \mathrm{P}^{q} \cong \mathrm{P}^{r} \text { (as } \Delta_{n} \text {-right modules). }
$$

In 1989, the first decomposition of $\Delta_{n}$ into principle indecomposable submodules was discovered and analyzed by Garsia and Reutenauer [6]. In the following we shall consider briefly its connection with the decomposition $\left\{\Lambda^{q} \mid q \vdash n\right\}$. For all $q \vDash n$, let

$$
I_{q}:=\sum_{r \models q} \frac{(-1)^{|r|+|q|}}{\prod_{i}\left|r\left(q_{i}\right)\right|} \Xi^{r} .
$$

Then the elements $\left(\prod_{j \in \underline{n}}\left(a_{j}(q) !\right)\right)^{-1} I_{q}$ are idempotents and form a $K$-basis of $\Delta_{n}$. If $q, s \models n$, then $I_{q} \equiv I_{s} \bmod \operatorname{Rad} \Delta_{n}$ if and only if $q \approx s$. Furthermore,

$$
\tilde{\Lambda}^{q}:=\left\langle I_{\dot{q}} \mid \dot{q} \in \mathbb{N}^{*}, \dot{q} \approx q\right\rangle_{K}
$$

is an indecomposable left ideal of $\Delta_{n}$ which is generated by the idempotent

$$
E_{q}:=\frac{1}{|q| !} \sum_{\dot{q} \approx q} I_{\dot{q}},
$$

and

$$
\Delta_{n}=\bigoplus_{q \vdash n} \tilde{\Lambda}^{q} \quad \text { for all } n \in \mathbb{N}
$$


These results were obtained in [6, Theorem 3.4, Theorem 4.2, Theorem 4.3]. (In the context of [6], where products of permutations are read from right to left, $\tilde{\Lambda}^{q}$ is, of course, a right ideal.) By the Krull-Schmidt theorem there exists a permutation $\sigma$ of the set of all partitions of $n$ such that $\Lambda^{q \sigma} \cong \tilde{\Lambda}^{q}$ as $\Delta_{n}$-modules, for all $q \vdash n$. By (8) and (2), $\sigma$ is uniquely determined. We shall prove that $\sigma=$ id, by means of the combinatorial Proposition 1.4. If $q, s \in \mathbb{N}^{*}$ such that $s \vDash q=q_{1} \cdots q_{k}$ and $c=\left(c_{1}, \ldots, c_{k}\right)$ is a $k$-tuple of positive integers such that $c_{j} \leq\left|s(q)_{j}\right|$ for all $j \in \underline{k}$, we put $I_{q, c}(s):=\prod_{j \in k}\left(s(q)_{j}\right)_{c_{j}}$. Then $I_{q}(s):=I_{q,(1, \ldots, 1)}(s)$ is the product of the initial letters of the words $s(q)_{j}$, and $F_{q}(s):=I_{q,\left(\left|s(q)_{1}\right| \ldots .,\left|s(q)_{k}\right|\right)}(s)$ is the product of the final letters of the words $s(q)_{j}$.

PROPOSITION 1.4. Let $q, s \in \mathbb{N}^{*}$ such that $s \models q=q_{1} \cdots q_{k}$. Let $s^{(1)}, \ldots, s^{(m)}$ be the compositions of $q$ such that $s^{(i)}(q)_{j} \approx s(q)_{j}$ for all $j \in \underline{k}, i \in \underline{m}$. Let $C$ be the set of all $k$-tuples $c=\left(c_{1}, \ldots, c_{k}\right)$ of positive integers such that $c_{j} \leq\left|s(q)_{j}\right|$ for all $j \in \underline{k}$. Then $m \prod_{j \in \underline{k}} q_{j}=\prod_{j \in \underline{k}}\left|s(q)_{j}\right| \sum_{i \in \underline{m}} I_{q, c}\left(s^{(i)}\right)$ for all $c \in C$.

PROOF. Let $f: C \times \underline{m} \rightarrow \mathbb{N}^{k},(c, i) \mapsto\left(s^{(i)}(q)_{1}\right)_{c_{1}}\left(s^{(i)}(q)_{2}\right)_{c_{2}} \cdots\left(s^{(i)}(q)_{k}\right)_{c_{k}}$. For any $c \in C, w \in \mathbb{N}^{k}$, we put $a(c, w):=|\{i \mid i \in \underline{m}, f(c, i)=w\}|$. If $\sigma_{j} \in S_{\left|s(q)_{j}\right|}$ for all $j \in \underline{k}, \sigma:=\left(\sigma_{1}, \ldots, \sigma_{k}\right)$, we put $\sigma s^{(i)}:=\sigma_{1} s^{(i)}(q)_{1} \cdots \sigma_{k} s^{(i)}(q)_{k}$. Then $\left\{s^{(1)}, \ldots, s^{(m)}\right\}=\left\{\sigma s^{(1)}, \ldots, \sigma s^{(m)}\right\}$, and therefore there exists a permutation $\sigma^{*} \in S_{m}$ such that $\sigma s^{(i)}=s^{\left(i \sigma^{*}\right)}$ for all $i \in \underline{m}$. Thus we have

$$
f\left(\left(c_{1} \sigma_{1}, \ldots, c_{k} \sigma_{k}\right), i\right)=f\left(c, i \sigma^{*}\right) \quad \text { for all } c \in C, i \in \underline{m} .
$$

This implies

$$
a(c, w)=a\left(c^{\prime}, w\right) \text { for all } c, c^{\prime} \in C, w \in \mathbb{N}^{k},
$$

as we may find permutations $\sigma_{j} \in S_{\left|s(q)_{j}\right|}$ such that $c_{j} \sigma_{j}=c_{j}^{\prime}$, and $\mid\{i \mid i \in \underline{m}$, $f(c, i)=w\}|=|\left\{i \mid i \in \underline{m}, f\left(c, i \sigma^{*}\right)=w\right\} \mid$. It follows that

$$
\begin{aligned}
m \prod_{j \in \underline{k}} q_{j} & =\sum_{i \in \underline{m}} \prod_{j \in \underline{k}} \operatorname{sum}\left(s^{(i)}(q)_{j}\right)=\sum_{i \in \underline{m}} \sum_{c \in C} \prod_{j \in \underline{k}}\left(s^{(i)}(q)_{j}\right)_{c_{j}}=\sum_{c \in C} \sum_{i \in \underline{m}} \prod_{j \in \underline{k}} f(c, i)_{j} \\
& =\sum_{c \in \mathcal{C}} \sum_{w \in \mathbb{N}^{k}} a(c, w) \prod_{j \in \underline{k}} w_{j}=\sum_{w \in \mathbb{N}^{k}}|C| a\left(c_{0}, w\right) \prod_{j \in \underline{k}} w_{j}, \quad \text { by }(10),
\end{aligned}
$$

for any $c_{0} \in C$. As $|C|=\prod_{j \in \underline{k}}\left|s(q)_{j}\right|$, this proves our claim.

PROPOSITION 1.5. Let $n$ be a positive integer and $q \vDash n$. Then

(a) $v_{q} \equiv E_{q} \equiv\left(\prod_{j \in \underline{n}}\left(a_{j}(q) !\right)\right)^{-1} I_{q} \bmod \operatorname{Rad} \Delta_{n}$,

(b) $\Lambda^{q} \cong \tilde{\Lambda}^{q}$ as $\Delta_{n}$-modules. 
ProOF. Let $q=q_{1} \cdots q_{k}$. The number of all $\dot{q} \models n$ such that $\dot{q} \approx q$ is $\left(\begin{array}{c}k \\ a_{1}(q), \ldots, a_{n}(q)\end{array}\right)$. This yields the second congruence in (a). By $[4,(10)]$,

$$
\begin{gathered}
v_{q} \equiv \sum_{r \vdash n} \sum_{r \approx r^{\prime} \mid=q} \frac{(-1)^{\left|r^{\prime}\right|+k} F_{q}\left(r^{\prime}\right)}{q ?} \Xi^{r}, \\
E_{q} \equiv \sum_{r \vdash n} \frac{1}{k !} \sum_{r \approx r^{\prime}=q^{\prime} \approx q} \frac{(-1)^{|r|+k}}{\prod_{i \in \underline{k}}\left|r^{\prime}\left(q^{\prime}\right)_{i}\right|} \Xi^{r}=\sum_{r \vdash n} \frac{1}{\prod_{j \in \underline{n}}\left(a_{j}(q) !\right)} \sum_{r \approx r^{\prime}=q} \frac{(-1)^{|r|+k}}{\prod_{i \in \underline{k}}\left|r^{\prime}(q)_{i}\right|} \Xi^{r}
\end{gathered}
$$

modulo $\operatorname{Rad} \Delta_{n}$. Let $r \vdash n$ such that $r \sim q$. For any $s \models q$ such that $r \approx s$ we put $[s]:=\left\{r^{\prime} \mid r^{\prime} \models q, r^{\prime}(q)_{j} \approx s(q)_{j}\right.$ for all $\left.j \in \underline{k}\right\}$. Then $\{[s] \mid r \approx s \vDash q\}$ is a partition of the set of all compositions of $q$ which are associate to $r$. Applying Proposition 1.4 to each class $[s]$ we obtain

$$
\sum_{r \approx r^{\prime} \neq q} \frac{\prod_{j \in \underline{k}} q_{j}}{\prod_{j \in \underline{k}}\left|r^{\prime}(q)_{j}\right|}=\sum_{r \approx r^{\prime} \vDash q} F_{q}\left(r^{\prime}\right)
$$

from which we conclude the first congruence in (a). By (a), $\Lambda^{q}+\operatorname{Rad} \Delta_{n}=\tilde{\Lambda}^{q}+$ $\operatorname{Rad} \Delta_{n}$, hence $\Lambda^{q} / \operatorname{Rad} \Lambda^{q} \cong \tilde{\Lambda}^{q} / \operatorname{Rad} \tilde{\Lambda}^{q}$ as $\Delta_{n}$-modules which implies (b) ([5, 45.7]).

Two different descriptions of the irreducible representations of $\Delta_{n}$ may be found in $[1,3$.$] and in [3, (50)]. If q, r \vDash n$, we write $C^{q}$ for the conjugacy class of all permutations of cycle structure $q$, and $\xi^{r}\left(C^{q}\right)$ for the value of any element of $C^{q}$ under the Young character $\xi^{r}([9,4.3])$. Then the linear mappings $c^{q}: \Delta_{n} \rightarrow K$ such that $c^{q}\left(\Xi^{r}\right)=\xi^{r}\left(C^{q}\right)$ for all $r \models n$ constitute the set of all irreducible characters of $\Delta_{n}([3$, (50)]). (Obviously, $q \approx \dot{q}$ implies that $C^{q}=C^{\dot{q}}$.) On the other hand, we conclude from $[4,1.3]$ that, for all $r, q \models n$,

$$
\Xi^{r} \omega_{q} \equiv \bar{m}_{q}^{r} \omega_{q} \bmod \operatorname{Rad} \Delta_{n},
$$

where $\bar{m}_{q}^{r}$ is the cardinality of the set $\overline{\mathscr{M}}_{q}^{r}$ of all matrices over $\mathbb{N}_{0}$ such that the sum of all rows is $q$, the sum of all columns is $r$, and each column contains a unique non-zero element. Hence the linear mappings $c_{*}^{q}: \Delta_{n} \rightarrow K$ such that $c_{*}^{q}\left(\Xi^{r}\right)=\bar{m}_{q}^{r}$ for all $r \vDash n$ also constitute the set of all irreducible characters of $\Delta_{n}$. A closer look shows that this second description is essentially a short version of that given in $[1,3$.$] .$ (Clearly, $q \approx \dot{q}$ implies that $c_{*}^{q}=c_{*}^{\dot{q}}$.) We show

Proposition 1.6. Let $n$ be a positive integer. Then $c^{q}=c_{*}^{q}$ for all $q \models n$.

PROOF. Let $q=q_{1} \cdots q_{k}, r=r_{1} \cdots r_{l} \vDash n$. We have to show that

$$
\xi^{r}\left(C^{q}\right)=\bar{m}_{q}^{r} .
$$


Let $Y$ be a Young subgroup of $S_{n}$ of type $S_{r_{1}} \times \cdots \times S_{r_{i}}$, and let $R_{1}, \ldots, R_{l}$ be the orbits of $Y$ in $\underline{n},\left|R_{i}\right|=r_{i}$ for all $i \in \underline{l}$. Then $\mathscr{S}:=\left\{\sigma\left|\sigma \in S_{n}, \sigma\right|_{R_{i}}\right.$ is increasing for all $i \in \underline{l}$ \} is a right transversal of $Y$ in $S_{n}$. Let $\pi \in C^{q}$, and let $Q_{1}, \ldots, Q_{k}$ be the orbits of $\pi$ in $\underline{n},\left|Q_{i}\right|=q_{i}$ for all $i \in \underline{k}$. If $\sigma \in \mathscr{S}$, then $Y \sigma \pi=Y \sigma$ if and only if $\sigma \in \mathscr{T}$, where $\mathscr{T}$ is the set of all $\tau \in \mathscr{S}$ such that $R_{i} \tau$ is a union of some of the sets $Q_{j}$. Hence $\xi^{r}\left(C^{q}\right)=\xi^{r}(\pi)=|\mathscr{T}|$. We now define a bijection of $\overline{\mathscr{M}}_{q}^{r}$ onto $\mathscr{T}$ : If $A=\left(a_{i j}\right) \in \overline{\mathscr{M}}_{q}^{r}$, then $\left|\bigcup\left\{Q_{j} \mid a_{i j} \neq 0\right\}\right|=r_{i}$, for all $i \in \underline{l}$. Therefore there exists a unique permutation $\sigma \in \mathscr{S}$ such that $R_{i} \sigma=\bigcup\left\{Q_{j} \mid a_{i j} \neq 0\right\}$ for all $i \in \underline{l}$. The mapping $A \mapsto \sigma$ is the desired bijection.

The foregoing proposition shows that $\left(\bar{m}_{q}^{r}\right)_{q, r \vdash n}$ is the table of the Young characters of $S_{n}$. It is remarkable that the numbers $\bar{m}_{q}^{r}$ are also given through the following formula:

PROPOSITION 1.7. Let $n$ be a positive integer, $q, r \vDash n$. Then

$$
\bar{m}_{q}^{r}=q ? \sum_{q \approx \dot{q} \models r} \frac{1}{\mathrm{psp}(\dot{q}, r)},
$$

where $\operatorname{psp}(w)$ is the product of the successive partial sums $w_{1}, w_{1}+w_{2}, w_{1}+w_{2}+$ $w_{3}, \ldots$ of the initial segments of the word $w \in \mathbb{N}^{*}$, and $\operatorname{psp}(\dot{q}, r)$ is the product (in $\mathbb{N}$ ) of the numbers $\operatorname{psp}\left(\dot{q}(r)_{i}\right)(\dot{q} \vDash r)$.

ProOF. By (3) and (11),

$$
\Xi^{r} \equiv \sum_{q \vdash n} \bar{m}_{q}^{r} \nu_{q}=\sum_{q \vdash n} \frac{1}{q ?} \bar{m}_{q}^{r} \omega_{q} \bmod \operatorname{Rad} \Delta_{n} .
$$

Furthermore, $\omega_{s}=(-1)^{|s|} \sum_{r k s}(-1)^{|r|} F_{s}(r) \Xi^{r}$ for all $s \models n$, by [4, 1.2]. From [7, Proposition 4.5] we may read off the inverse of the matrix of scalar coefficients of this system of equations, and we conclude that

$$
\Xi^{r}=\sum_{s \models r} \frac{1}{\operatorname{psp}(s, r)} \omega_{s} \equiv \sum_{q \vdash n} \sum_{q \approx \dot{\sim} \models r} \frac{1}{\operatorname{psp}(\dot{q}, r)} \omega_{q} \bmod \operatorname{Rad} \Delta_{n} .
$$

Comparing with (13), we obtain the asserted formula.

By [12], there is an algebra epimorphism $c$ of $\Delta_{n}$ onto the $K$-algebra $C l\left(S_{n}\right)$ of all class functions of $S_{n}$ with the property that $c\left(\Xi^{r}\right)=\xi^{r}$ for all $r \vDash n$. If $\eta$ is a primitive idempotent of $\Delta_{n}$, then $c(\eta)$ is a primitive idempotent of $C l\left(S_{n}\right)$. Therefore, there exists a conjugacy class $C$ of $S_{n}$ such that $c(\eta)$ is the characteristic function $\operatorname{char}_{C}$ of $C$. More precisely, we prove the following 
PROPOSITION 1.8. Let $n$ be a positive integer, $q \vdash n$, and let $\eta$ be an idempotent of $\Delta_{n}$ such that $\eta \equiv v_{q} \bmod \operatorname{Rad} \Delta_{n}$. Then $c(\eta)=\operatorname{char}_{C^{q}}$.

Proof. Without loss of generality we may assume that $\eta=v_{q}$. We know that there exists a partition $\tilde{q}$ of $n$ such that $c\left(v_{q}\right)=\operatorname{char}_{C^{\dot{q}}}$. We have to show that $q=\tilde{q}$. For any $r \vdash n$ we have

$$
\begin{aligned}
\bar{m}_{\tilde{q}}^{r} \operatorname{char}_{C^{\tilde{q}}} & =\xi^{r}\left(C^{\tilde{q}}\right) \operatorname{char}_{C^{\tilde{q}}} & \text { by (12) } \\
& =\xi^{r} \operatorname{char}_{C^{\bar{q}}}=c\left(\Xi^{r} v_{q}\right)=c\left(\bar{m}_{q}^{r} v_{q}\right) & \text { by (11) } \\
& =\bar{m}_{q}^{r} \operatorname{char}_{C^{\bar{q}} .} . &
\end{aligned}
$$

Therefore, $\bar{m}_{\tilde{q}}^{r}=\bar{m}_{q}^{r}$ for all $r \vdash n$. In particular, $\bar{m}_{\bar{q}}^{q}=\bar{m}_{q}^{q} \neq 0$ and $\bar{m}_{q}^{\bar{q}}=\bar{m}_{\tilde{q}}^{\bar{q}} \neq 0$. Hence $\tilde{q} \sim q \sim \tilde{q}$, that is, $q=\tilde{q}$.

\section{Lower Loewy factors and Poincaré-Birkhoff-Witt bases}

We now consider the free associative $K$-algebra of countable rank $K \mathbb{N}^{*}$, the semigroup algebra of $\mathbb{N}^{*}$ over $K$. For every $n \in \mathbb{N}_{0}$ let $D_{n}$ be the $K$-linear span in $K \mathbb{N}^{*}$ of all compositions of $n$. Then

$$
K \mathbb{N}^{*}=\bigoplus_{n \geq 0} D_{n}
$$

The $K$-linear mapping $\omega: K \mathbb{N}^{*} \rightarrow \Delta$ such that $q \omega=\omega_{q}$ for all $q \in \mathbb{N}^{*}$ is a vector space isomorphism, and $D_{n} \omega=\Delta_{n}$ for all $n \in \mathbb{N}_{0}$. (It should be noted that the vector space isomorphism $\alpha: \Delta \rightarrow K \mathbb{N}^{*}$ considered in [7, 5.1] is not the inverse of $\omega$.) For every $q \in \mathbb{N}^{*}$, the multihomogeneous component $\left\langle\dot{q} \mid \dot{q} \in \mathbb{N}^{*}, \dot{q} \approx q\right\rangle_{K}$ of $K \mathbb{N}^{*}$ is mapped onto $\Lambda^{q}$. This shows that the statement (ii) in our introduction holds; statement (i), however, is less obvious. We first give a description of the lower central series of an arbitrary associative $K$-algebra $A$ in terms of a system of generators of $A$.

The standard Lie product $\circ$ in $A$ is defined by

$$
a \circ b:=a b-b a \quad(a, b \in A) .
$$

Inductively, we set $a[1] \circ \cdots \circ a[m]:=(a[1] \circ \cdots \circ a[m-1]) \circ a[m]$ for all $a[1], \ldots, a[m] \in A, m \geq 2$. The following proposition will be useful later on:

PROPOSITION 2.1. Let $A$ be an associative $K$-algebra, $j_{1}, \ldots, j_{l} \in \mathbb{N}, h:=j_{1}+$ $\cdots+j_{l}$, and let $u[i] \in \gamma^{\left(j_{i}\right)}(A)$ for all $i \in \underline{l}$. Then

$$
u[1] \cdots u[l]-u[1 \sigma] \cdots u[l \sigma] \in \gamma^{(h+1)}(A) \quad \text { for all } \sigma \in S_{l} .
$$


Proof. We may assume that $\sigma=(i, i+1), i \in l-1$, as $S_{l}$ is generated by the transpositions of this kind. By $[8,3.4], u[i] \circ u[i+1] \in \gamma^{\left(j_{i}+j_{i+1}+1\right)}(A)$, hence $u[1] \cdots u[l]-u[1 \sigma] \cdots u[l \sigma]=u(1) \cdots u[i-1](u[i] \circ u[i+1]) u[i+2] \cdots u[l] \epsilon$ $\gamma^{(h+1)}(A)$, by $[8,3.3]$.

For any subset $X$ of $A$ and $j \in \mathbb{N}_{0}$ let $R^{(j), X}$ be the $K$-linear span of the elements $\left(x_{1} \circ \cdots \circ x_{i_{1}}\right)\left(x_{i_{1}+1} \circ \cdots \circ x_{i_{1}+i_{2}}\right) \cdots\left(x_{i_{1}+\cdots+i_{k-j+1}+1} \circ \cdots \circ x_{k}\right)$, where $x_{i} \in X$ and $j<k=i_{1}+\cdots+i_{k-j}$. That is, the generators of $R^{(j), X}$ arise from the products of length $>j$ over $X$ by inserting $j$ o's and bracketing accordingly. Then $R^{(j), X}$ is an ideal of the subalgebra of $A$ generated by $X$, and $R^{(j), X} \subseteq \gamma^{(j)}(A)$ by $[8,3.3]$.

PROPOSITION 2.2. If $X$ is a system of generators of an associative $K$-algebra $A$, then $R^{(j), X}=\gamma^{(j)}(A)$ for all $j \in \mathbb{N}_{0}$.

PROOF. by induction on $j$. The inclusion $\gamma^{(j)}(A) \subseteq R^{(j), X}$ holds for $j=0$ by our hypothesis on $X$. Now let $j>0$ and assume that $\gamma^{(j-1)}(A) \subseteq R^{(j-1), X}$. It suffices to show the following:

$$
\text { If } v \text { is a product of elements of } X \text {, then } \gamma^{(j-1)}(A) \circ v \subseteq R^{(j), X} \text {. }
$$

Let $b \in \gamma^{(j-1)}(A)$. By means of our inductive hypothesis we may assume that

$$
b=\left(x_{1} \circ \cdots \circ x_{i_{1}}\right)\left(x_{i_{1}+1} \circ \cdots \circ x_{i_{1}+i_{2}}\right) \cdots\left(x_{i_{1}+\cdots i_{1-1}+1} \circ \cdots \circ x_{i_{1}+\cdots+i_{i}}\right),
$$

where $i_{1}+\cdots+i_{l}-l=j-1$. If $v \in X$, the derivation rule

$$
\left(y_{1} \cdots y_{n}\right) \circ z=\sum_{k=1}^{n} y_{1} \cdots y_{k-1}\left(y_{k} \circ z\right) y_{k+1} \cdots y_{n} \quad \text { for all } y_{1}, \ldots, y_{n}, z \in A
$$

implies that $b \circ v \in R^{(j), X}$. If $v_{1}, \ldots, v_{n} \in X$ and $v=v_{1} \cdots v_{n}$, this in turn yields $b \circ v=\sum_{k=1}^{n} v_{1} \cdots v_{k-1}\left(b \circ v_{k}\right) v_{k+1} \cdots v_{n} \in R^{(j), X}$. This shows (15), and the proposition is proved.

We now apply Proposition 2.2 to the $K$-algebra $A=K \mathbb{N}^{*}$ and put $X:=\mathbb{N}$. Then for all $j \in \mathbb{N}_{0}$ we obtain $\gamma^{(j)}\left(K \mathbb{N}^{*}\right)=R^{(j), \mathbb{N}}=\bigoplus_{n \in \mathbb{N}_{0}}\left(R^{(j), \mathbb{N}} \cap D_{n}\right)$, and $R^{(j)}\left(D_{n}\right)=$ $R^{(j), N} \cap D_{n}$ is mapped by $\omega$ onto $\left(\operatorname{Rad} \Delta_{n}\right)^{j}$, by the main result of [4]. (For the definition of $R^{(j)}\left(D_{n}\right)$, see the introduction of [4]). Since $(\operatorname{Rad} \Delta)^{j}=\bigoplus_{n \in \mathbb{N}_{0}}\left(\operatorname{Rad} \Delta_{n}\right)^{j}$ for all $j \in \mathbb{N}_{0}$, we have thus shown the following

THEOREM 2.1. $\left(\gamma^{(j)}\left(K \mathbb{N}^{*}\right)\right) \omega=(\operatorname{Rad} \Delta)^{j}$ for all $j \in \mathbb{N}_{0}$.

For any composition $q$ of a positive integer $n$, we now study the composition structure of $\Lambda^{q}$ and $\mathrm{P}^{q}$. A composition $s$ of $r=r_{1} \cdots r_{l} \in \mathbb{N}^{*}$ is called power-free if $\left|s(r)_{i}\right|>1$ implies that $s(r)_{i}$ has at least two different letters, for every $i \in l$. 
THEOREM 2.2. Let $n$ be a positive integer and $q, r \vdash n$. For every $j \in \mathbb{N}_{0}$, the following statements are equivalent:

(i) $M^{r}$ is isomorphic to a composition factor of the $\Delta_{n}$-module $\operatorname{Rad}^{(j)} \Lambda^{q} /$ $\operatorname{Rad}^{(j+1)} \Lambda^{q}$,

(ii) $j=|q|-|r|$, and there exists a power-free composition of $r$ which is associate to $q$,

(iii) $M^{q}$ is isomorphic to a composition factor of the $\Delta_{n}$-module $\operatorname{Rad}^{(j)} \mathrm{P}^{r} /$ $\operatorname{Rad}^{(j+1)} \mathrm{P}^{\prime}$.

As a first step of the proof, we shall show that (i) implies the first and part of the second condition in (ii). For any $q, r \vDash n$ we show

(i) implies that $j=|q|-|r|$ and $q \sim r$.

For $j=0$, this follows from (7). Let $j>0$. By $[4,2.4]$,

$$
\operatorname{Rad}^{(j)} \Lambda^{q} / \operatorname{Rad}^{(j+1)} \Lambda^{q}=\left(\sum_{\substack{q \vdash s+n \\|q|-|s|=1}} \operatorname{Rad}^{(j-1)} \Lambda^{s}\right) \omega_{q} /\left(\sum_{\substack{q \nmid s\} n \\|q|-|s|=1}} \operatorname{Rad}^{(j)} \Lambda^{s}\right) \omega_{q} .
$$

Hence there exists $s \vdash n$ such that $q \vdash s,|q|-|s|=1$, and $M^{r}$ is a composition factor of the $\Delta_{n}$-module $\operatorname{Rad}^{(j-1)} \Lambda^{s} / \operatorname{Rad}^{(j)} \Lambda^{s}$. Inductively we have $j-1=|s|-|r|$ and $s \sim r$. The claim follows.

We will continue the proof of Theorem 2.2 at a later stage as we need some more preparations.

PROPOSITION 2.3. Let $n$ be a positive integer, $q, r \models n,|q| \geq|r|, h:=|q|-|r|$. Let $\eta$ be an idempotent of $\Delta_{n}$ such that $\eta \equiv v_{r} \bmod \operatorname{Rad} \Delta_{n}$, and let $\Lambda$ be a left ideal of $\Delta_{n}$ isomorphic to $\Lambda^{q}$. Then

(a) neither $\operatorname{Rad}^{(h+1)} \Lambda$ nor $\Lambda / \operatorname{Rad}^{(h)} \Lambda$ has a composition factor isomorphic to $M^{r}$,

(b) $\eta \Lambda \cap \operatorname{Rad}^{(h+1)} \Lambda=0$ and $\eta \Lambda \subseteq \operatorname{Rad}^{(h)} \Lambda$,

(c) $\eta \Lambda+\operatorname{Rad}^{(h+1)} \Lambda / \operatorname{Rad}^{(h+1)} \Lambda$ is the homogeneous $M^{r}$-component of $\operatorname{Rad}^{(h)} \Lambda /$ $\operatorname{Rad}^{(h+1)} \Lambda$,

(d) $\operatorname{dim}_{K} \eta \Lambda$ is the multiplicity of $M^{r}$ in a composition series of $\Lambda$.

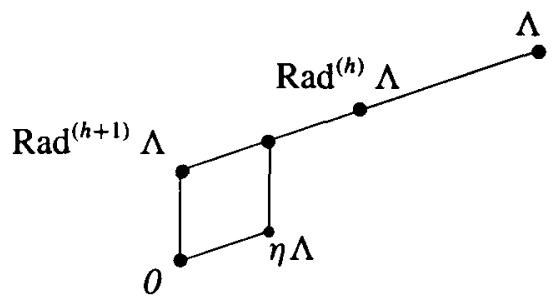


PROOF. (a) is an immediate consequence of (16). By (a) and Proposition 1.1(a), every composition factor of $\operatorname{Rad}^{(h+1)} \Lambda$ and of $\Lambda / \operatorname{Rad}^{(h)} \Lambda$, and therefore these modules themselves, are annihilated by the idempotent $\eta \equiv \nu_{r} \bmod \operatorname{Rad} \Delta_{n}$. It follows (b) as $\eta \Lambda \cap \operatorname{Rad}^{(h+1)} \Lambda=\eta \operatorname{Rad}^{(h+1)} \Lambda$. By the second part of (b), $\eta \Lambda+$ $\operatorname{Rad}^{(h+1)} \Lambda / \operatorname{Rad}^{(h+1)} \Lambda=\eta\left(\operatorname{Rad}^{(h)} \Lambda / \operatorname{Rad}^{(h+1)} \Lambda\right)$, hence (c). Finally, (d) is a special case of Proposition 1.1 (b).

In particular $\left(\eta:=v_{r}, \Lambda:=\Lambda^{q}\right)$, we set

$$
\Lambda^{r, q}:=\Lambda^{r} \Lambda^{q}+\operatorname{Rad}^{(h+1)} \Lambda^{q}\left(=v_{r} \Lambda^{q}+\operatorname{Rad}^{(h+1)} \Lambda^{q}\right) .
$$

A preliminary consequence of Proposition 2.3 (c) is the equation

$$
\Lambda^{q}=\sum_{r+n} v_{r} \Lambda^{q}
$$

Our next aim is to exhibit a basis of a vector space complement of $\operatorname{Rad}^{(h+1)} \Lambda^{q}$ in $\Lambda^{r, q}$. In this manner we shall obtain bases of $\Delta_{n}$ which are compatible with the Loewy series of every left ideal $\Lambda^{q}$ (see Theorem 2.3 (b)). Moreover, thanks to Proposition 2.3 (d), this will lead to an explicit formula for the Cartan invariant $c_{r, q}$, that is, for the multiplicity of $M^{r}$ in a composition series of $\Lambda^{q}$. With respect to the lexicographically decreasing order of the set of all partitions of $n$, we obtain from (16) and (4) the following:

(18) The Cartan matrix $\left(c_{r, q}\right)_{r, q \vdash n}$ is upper triangular, and $c_{q, q}=1$ for all $q \vdash n$.

Let $\mathbb{N}^{(*)}$ be the free magma over the set $\mathbb{N}$ and $K \mathbb{N}^{(*)}$ the free $K$-algebra generated by $\mathbb{N}$. Then the identity mapping of $\mathbb{N}$ extends uniquely to a magma epimorphism $\alpha$ of $\mathbb{N}^{(*)}$ onto $\mathbb{N}^{*}$ and to an algebra homomorphism $\lambda$ of $K \mathbb{N}^{(*)}$ into the Lie algebra $\left(K \mathbb{N}^{*},+, o\right)$. The image $L:=\left(K \mathbb{N}^{(*)}\right) \lambda$ is a free Lie algebra of countable rank, by $[2,2.3 .3]$. A bracketing of a subset $B$ of $\mathbb{N}^{*}$ is a mapping $\beta$ of $B$ in $\mathbb{N}^{(*)}$ such that $w \beta \alpha=w$ for all $w \in B$. The special bracketing $\beta_{l}$ of $\mathbb{N}^{*}$ defined by $w \beta_{l}:=\left(\left(\ldots\left(\left(w_{1} w_{2}\right) w_{3}\right) \cdots\right) w_{m}\right)$ (where $\left.m=|w|\right)$ is called left-normed. We put

$$
w^{\circ}:=w \beta_{l} \lambda \quad \text { for all } w \in \mathbb{N}^{*} .
$$

The linear extension of $\beta_{l} \lambda$ to $K \mathbb{N}^{*}$ is known as the Dynkin mapping. It is easily seen that $L$ is generated as a $K$-vector space by the set $\mathbb{N}^{*} \beta_{l} \lambda$, but no subset $B$ of $\mathbb{N}^{*}$ is known for which $B \beta_{l} \lambda$ is a $K$-basis of $L$.

A pair $(B, \beta)$ is called basic if $\beta$ is a bracketing of $B \subseteq \mathbb{N}^{*}$ such that $\beta \lambda$ is a bijective mapping of $B$ onto a $K$-basis of $L$. For example, $B$ may be chosen as the set of all Lyndon words in $\mathbb{N}^{*}$, and $\beta$ be defined via the standard Lyndon factorization (see $[10,5.3])$. If $(B, \beta)$ is any basic pair, we set

$$
w^{\square}:=w \beta \lambda \quad \text { for all } w \in B .
$$


Obviously, $w^{\square}$ is contained in the $K$-linear span of $\left\{\dot{w}^{\circ} \mid \dot{w} \approx w\right\}$. In particular,

$$
w^{\square} \in \gamma^{(m-1)}\left(K \mathbb{N}^{*}\right) \quad \text { for all } w \in B \text { such that }|w|=m .
$$

If $\leq$ is any linear order of $B$, the set

$$
\mathfrak{B}(B, \beta, \leq):=\left\{w[1]^{\square} \cdots w[j]^{\square} \mid j \in \mathbb{N}_{0}, w[i] \in B, w[1] \geq \cdots \geq w[j]\right\}
$$

is a $K$-basis of $K \mathbb{N}^{*}$, by the Poincare-Birkhoff-Witt theorem [2, 2.5.2]. A $K$-basis of $K \mathbb{N}^{*}$ of this kind is called a Poincaré-Birkhoff-Witt basis.

Now let $\mathfrak{B}=\mathfrak{B}(B, \beta, \leq)$ be a Poincaré-Birkhoff-Witt basis of $K \mathbb{N}^{*}$. Then $\mathfrak{B} \subseteq \bigcup_{n \in \mathbb{N}_{0}} D_{n}$, so that (14) implies

$$
\mathfrak{B} \cap D_{n} \text { is a } K \text {-basis of } D_{n} \text {, }
$$$$
\left(\mathfrak{B} \cap D_{n}\right) \omega \text { is a } K \text {-basis of } \Delta_{n} \text {, }
$$

for any $n \in \mathbb{N}_{0}$. For all $q \in \mathbb{N}^{*}$ put

$$
\mathfrak{B}_{q}:=\left\{w[1]^{\square} \cdots w[j]^{\square} \mid j \in \mathbb{N}_{0}, w[i] \in B, w[1] \geq \cdots \geq w[j], w[1] \cdots w[j] \approx q\right\} .
$$

For any $n \in \mathbb{N}$ we have, by (21),

$$
\mathfrak{B}_{q} \omega \text { is a } K \text {-basis of } \Lambda^{q} \text {, for all } q \models n .
$$

Let $q, q^{\prime} \in \mathbb{N}^{*}$. Then $\mathfrak{B}_{q}=\mathfrak{B}_{q^{\prime}}$ if $q \approx q^{\prime}$, and $\mathfrak{B}_{q} \cap \mathfrak{B}_{q^{\prime}}=\emptyset$ if $q \not \approx q^{\prime}$. If $r \in \mathbb{N}^{*}$ and $l:=|r|$, put

$$
\begin{gathered}
\mathfrak{A}^{r}:=\left\{w[1]^{\square} \cdots w[l]^{\square} \mid w[i] \in B, w[i] \models r_{i}, w[1] \geq \cdots \geq w[l]\right\}, \\
\mathfrak{B}^{r}:=\bigcup_{r^{\prime} \approx r} \mathfrak{A}^{r^{\prime}}, \quad \mathfrak{B}_{q}^{r}:=\mathfrak{B}^{r} \cap \mathfrak{B}_{q} .
\end{gathered}
$$

Let $r, r^{\prime} \in \mathbb{N}^{*}$. Then $\mathfrak{B}^{r}=\mathfrak{B}^{r^{\prime}}$ if $r \approx r^{\prime}$, and $\mathfrak{B}^{r} \cap \mathfrak{B}^{r^{\prime}}=\emptyset$ if $r \not \approx r^{\prime}$. Thus for every $n \in \mathbb{N}$ we have

$$
\mathfrak{B} \cap D_{n}=\bigcup_{r \vdash n} \mathfrak{B}^{r}=\bigcup_{q \vdash n} \mathfrak{B}_{q}=\bigcup_{\substack{+\rightarrow n \\ q \vdash n}} \mathfrak{B}_{q}^{r},
$$

and these unions are disjoint. Furthermore, it is easily seen that

$$
\text { if } \mathfrak{B}_{q}^{r} \neq \emptyset \text {, then } q \sim r \text {. }
$$

Moreover, if $q \approx r$, then $\left|\mathfrak{B}_{q}^{r}\right|=1$. If $\leq$ extends the usual order of $\mathbb{N}$ and $q \approx r$, then $\mathfrak{B}_{q}^{r}=\{p\}$, where $p$ is the unique partition such that $q \approx p \approx r$. 
THEOREM 2.3. Let $n$ be a positive integer, $q, r \models n,|q| \geq|r|, h:=|q|-|r|$. Then

(a) $\left\langle\mathfrak{B}_{q}^{r}\right\rangle_{K} \omega$ is a vector space complement of $\operatorname{Rad}^{(h+1)} \Lambda^{q}$ in $\Lambda^{r, q}$,

(b) $\bigcup_{\substack{q|j| s|a+1\\| j|\leq|-j}} \mathfrak{B}_{q}^{s} \boldsymbol{\omega}$ is a $K$-basis of $\operatorname{Rad}^{(j)} \Lambda^{q}$, for all $j \in \mathbb{N}_{0}$.

PROOF. Let $l:=|r|$. We first show

$$
\omega_{r} \Lambda^{q} \subseteq\left\langle\omega_{\dot{q}(r)_{1}^{\circ} \cdots \dot{q}(r)_{i}^{\circ}} \mid q \approx \dot{q} \vDash r\right\rangle_{K} \subseteq \operatorname{Rad}^{(h+1)} \Lambda^{q}+\left\langle\mathfrak{B}_{q}^{r}\right\rangle_{K} \omega .
$$

The first inclusion follows directly from $[4,1.5]$. As for the second, let $\dot{q} \vDash r$ such that $\dot{q} \approx q$. For any $i \in \underline{l}$ let $c_{i, w} \in K$ such that $\dot{q}(r)_{i}^{\circ}=\sum_{w \in B, w \approx \dot{q}(r)_{i}} c_{i, w} w^{\square}$. Then

$$
\dot{q}(r)_{1}^{\circ} \cdots \dot{q}(r)_{l}^{\circ}=\sum c_{1, w[1]} \cdots c_{l, w[l]} w[1]^{\square} \cdots w[l]^{\square},
$$

where the sum ranges over all $l$-tuples $(w[1], \ldots, w[l])$ over $B$ such that $w[i] \approx \dot{q}(r)_{i}$. Clearly, for all these and for all $\sigma \in S_{l}$ we have $w[1 \sigma]^{\square} \cdots w[l \sigma]^{\square} \in\left\langle q^{\prime} \mid q^{\prime} \approx q\right\rangle_{K} \subseteq D_{n}$. Hence $w[1]^{\square} \cdots w[l]^{\square}-w[1 \sigma]^{\square} \cdots w[l \sigma]^{\square} \in \gamma^{(h+1)}\left(K \mathbb{N}^{*}\right) \cap D_{n}=R^{(h+1)}\left(D_{n}\right)$ by Proposition 2.1, (19), and Proposition 2.2. We may choose $\sigma$ such that $w[1 \sigma] \geq$ $\cdots \geq w[l \sigma]$, and therefore we have $w[1]^{\square} \cdots w[l]^{\square} \in R^{(h+1)}\left(D_{n}\right)+\left\langle\mathfrak{B}_{q}^{r}\right\rangle_{K}$.

Applying [4, 2.5 Main Theorem], we conclude that

$$
\omega_{\dot{q}(r)_{i}^{\circ \cdots} \dot{q}(r)_{i}^{\circ}} \in\left(\operatorname{Rad}^{(h+1)} \Delta_{n}+\left\langle\mathfrak{B}_{q}^{r}\right\rangle_{K} \omega\right) \cap \Lambda^{q}=\operatorname{Rad}^{(h+1)} \Lambda^{q}+\left\langle\mathfrak{B}_{q}^{r}\right\rangle_{K} \omega .
$$

This proves (25). As a consequence, we note that

$$
\operatorname{dim}_{K}\left(\omega_{r} \Lambda^{q}\right) \leq\left|\mathfrak{B}_{q}^{r}\right|,
$$

because $\omega_{r} \Lambda^{q} \cap \operatorname{Rad}^{(h+1)} \Lambda^{q}=v_{r} \Lambda^{q} \cap \operatorname{Rad}^{(h+1)} \Lambda^{q}=0$ by Proposition $2.3($ b).

From (2) and (17) we conclude that $\Delta_{n}=\sum_{q, r \vdash n} \omega_{r} \Lambda^{q}$. Hence, by (26), (23), and (20),

$$
2^{n-1}=\operatorname{dim}_{K} \Delta_{n} \leq \sum_{q, r \vdash n} \operatorname{dim}_{K}\left(\omega_{r} \Lambda^{q}\right) \leq \sum_{q, r+n}\left|\mathfrak{B}_{q}^{r}\right|=\left|\mathfrak{B} \cap D_{n}\right|=\operatorname{dim} D_{n}=2^{n-1} .
$$

Therefore, the inequality in (26) is an equality:

$$
\operatorname{dim}_{K}\left(\omega_{r} \Lambda^{q}\right)=\left|\mathfrak{B}_{q}^{r}\right|
$$

By Proposition 2.3(b) and (25), we have

$$
\Lambda^{r, q}=\operatorname{Rad}^{(h+1)} \Lambda^{q} \oplus v_{r} \Lambda^{q} \subseteq \operatorname{Rad}^{(h+1)} \Lambda^{q}+\left\langle\mathfrak{B}_{q}^{r}\right\rangle_{K} \omega
$$

from which we obtain (a), by means of (27).

Using Proposition 2.3 (b), (c), we conclude from (a) that

$$
\operatorname{Rad}^{(j)} \Lambda^{q}=\operatorname{Rad}^{(j+1)} \Lambda^{q} \oplus \bigoplus_{\substack{q|<+\leqslant n\\| s|=| q \mid-j}}\left\langle\mathfrak{B}_{q}^{s} \omega\right\rangle_{K} \text {, for all } j \in \mathbb{N}_{0} .
$$

Clearly, this implies (b). 
If $\eta$ is an idempotent $\neq 0$ of $\Lambda^{r}$, it is not true that one has $\eta \Lambda^{q}=\left\langle\mathfrak{B}_{q}^{r}\right\rangle_{K} \omega$ for an appropriate choice of $\mathfrak{B}$. For example, $\eta=\nu_{33}$ is the only idempotent $\neq 0$ of $\Lambda^{33}$, and $\nu_{33} \Lambda^{321}=\left\langle\omega_{3(201)}+\omega_{(201) 3}\right\rangle_{K} \nsupseteq \mathfrak{B}_{321}^{33} \omega$, for any Poincaré-Birkhoff-Witt basis $\mathfrak{B}$.

In view of Proposition 1.5 (b) and Proposition 1.1 (b), the statement (27) could also be derived from [6, Theorem 5.4]. We observe that $\left|\mathfrak{B}_{q}^{n}\right|$ is a necklace number ([11, (7.1.2)]), because $\mathfrak{B}_{q}^{n}=\left\{w^{\square} \mid q \approx w \in B\right\}$ is a $K$-basis of the homogeneous component $\left\langle\dot{q}^{\circ} \mid \dot{q} \approx q\right\rangle_{K}$ of the free Lie algebra $L$ over $\mathbb{N}$. By Witt's formula [13, Satz 3], its dimension is

$$
h_{q}:=\frac{1}{|q|} \sum_{d} \mu(d) \frac{\frac{|q|}{d} !}{\prod_{j}\left(\frac{a_{j}(q)}{d} !\right)},
$$

where the sum is taken over all common divisors $d$ of the numbers $a_{j}(q)=\mid\{i \mid i \leq$ $\left.|q|, q_{i}=j\right\} \mid, j \in \mathbb{N}$. The numbers $h_{w}\left(w \in \mathbb{N}^{*}\right)$ coincide with the special Cartan invariants $c_{\text {sum(w),w }}$. They will occur in a purely combinatorial formula for the Cartan invariants of $\Delta_{n}$ in general which we shall now obtain.

If $r \in \mathbb{N}^{*}, l:=|r|, s \vDash r$, we put $a_{w}(s(r)):=\left|\left\{i \mid i \in \underline{l}, s(r)_{i}=w\right\}\right|$ for all $w \in \mathbb{N}^{*}$. If, moreover, $r$ is a partition, then $s$ is called a partition of $r(s \vdash r)$ if the following two conditions hold:

for every $i \in \underline{l} \quad s(r)_{i} \vdash r_{i}$,

for every $i \in \underline{l-1}: \quad r_{i}=r_{i+1} \Longrightarrow s(r)_{i+1} \leq_{\operatorname{lex}} s(r)_{i}$

(where $\leq_{\text {lex }}$ is the lexicographic order of $\mathbb{N}^{*}$ ).

COROLLARY 2.1. Let $n$ be a positive integer, $q, r \vdash n$.

(a) The Cartan invariant $c_{r, q}$ is equal to $\sum_{q \approx \dot{q} \vdash r} \prod_{w \in \mathbb{N}^{*}}\left(\begin{array}{c}h_{w}+a_{w}(\dot{q}(r))-1 \\ a_{w}(\dot{q}(r))\end{array}\right)$. (Each of the products has only a finite number of factors $\neq 1$, because $a_{w}(\dot{q}(r))=0$ if $w$ is not one of the partitions $\dot{q}(r)_{i}$.)

(b) $M^{r}$ is isomorphic to a composition factor of $\Lambda^{q}$ if and only if there exists a power-free composition of $r$ which is associate to $q$.

PROOF. (a) For all $w \in \mathbb{N}^{*}$, we write $\bar{w}$ for the partition associate to $w$. By Proposition 2.3 (d) and (27), we have

$$
c_{r, q}=\left|\mathfrak{B}_{q}^{r}\right|
$$

for every Poincaré-Birkhoff-Witt basis $\mathfrak{B}=\mathfrak{B}(B, \beta, \leq)$. We choose an order $\leq$ of $B$ with the property that

(i) $\operatorname{sum}(v)<\operatorname{sum}(w) \Rightarrow v \leq w$,

(ii) $\operatorname{sum}(v)=\operatorname{sum}(w), \bar{v} \leq_{\text {lex }} \bar{w} \Rightarrow v \leq w$, 
for all $v, w \in B$, and prove (a) by counting $\mathfrak{B}_{q}^{r}$. Let $l:=|r|, B_{q}^{r}:=\{(w[1], \ldots, w[l]) \mid$ $\left.w[i] \in B, w[i] \vDash r_{i}, w[1] \geq \cdots \geq w[l], w[1] \cdots w[l] \approx q\right\}$. By (i), $\mathfrak{B}^{r}=\mathfrak{A}^{r}$, hence $\left|\mathfrak{B}_{q}^{r}\right|=\left|B_{q}^{r}\right|$. By (ii), $f:(w[1], \ldots, w[l]) \mapsto \overline{w[1]} \cdots \overline{w[l]}$ is a mapping of $B_{q}^{r}$ into $\left\{\dot{q} \mid \dot{q} \in \mathbb{N}^{*}, q \approx \dot{q} \vdash r\right\}=: P$.

For every partition $p \in \mathbb{N}^{*}$ we have $|\{w \mid w \in B, \bar{w}=p\}|=h_{p}$. Exploiting (i), (ii), and (1), a moment's reflection reveals that, for any $\dot{q} \in P$,

$$
\left|f^{-1}(\dot{q})\right|=\prod_{p}\left(\begin{array}{c}
h_{p}+a_{p}(\dot{q}(r))-1 \\
a_{p}(\dot{q}(r))
\end{array}\right),
$$

where $p$ ranges over the set $\left\{\dot{q}(r)_{1}, \ldots, \dot{q}(r)_{l}\right\}$. Now (a) follows. Furthermore, $\left|f^{-1}(\dot{q})\right|=0$ if and only if there exists a partition $p$ such that $a_{p}(\dot{q}(r)) \neq 0$ and $h_{p}=0$. This means that $\dot{q}(r)$ is not power-free. Therefore (a) shows that $c_{r, q} \neq 0$ if and only if there exists a power-free element $\dot{q} \in P$. This proves (b).

Now it is easy to obtain the statements corresponding to Corollary 2.1 for the right ideals $\mathrm{P}^{r}$.

COROLLARY 2.2. Let $n$ be a positive integer, $q, r \vdash n$.

(a) The multiplicity of $M^{q}$ in a composition series of $\mathrm{P}^{r}$ is equal to $c_{r, q}$.

(b) $M^{q}$ is isomorphic to a composition factor of $\mathrm{P}^{r}$ if and only if there exists a power-free composition of $r$ which is associate to $q$.

PROOF. (a) follows from Proposition 1.1 (b) and the equation $\mathrm{P}^{q} \nu_{q}=v_{r} \Delta_{n} v_{q}=v_{r} \Lambda^{q}$. By (a) and by Corollary 2.1 (b), both conditions in (b) are equivalent to $c_{r, q} \neq 0$.

COROLLARY 2.3. Let $n$ be a positive integer. Then

$$
\sum_{r \vdash n} \sum_{q \approx \dot{q} \vdash r} \prod_{w \in \mathbb{N}^{*}}\left(\begin{array}{c}
h_{w}+a_{w}(\dot{q}(r))-1 \\
a_{w}(\dot{q}(r))
\end{array}\right)=\operatorname{dim}_{K} \Lambda^{q}=\left(\begin{array}{c}
|q| \\
a_{1}(q), \ldots, a_{n}(q)
\end{array}\right)
$$

for all $q \vDash n$,

$$
\sum_{q \vdash n} \sum_{q \approx \dot{q} \vdash r} \prod_{w \in \mathbb{N}^{*}}\left(\begin{array}{c}
h_{w}+a_{w}(\dot{q}(r))-1 \\
a_{w}(\dot{q}(r))
\end{array}\right)=\operatorname{dim}_{K} \mathrm{P}^{r}=\prod_{d=1}^{n}\left(\begin{array}{c}
l_{d}+a_{d}(r)-1 \\
a_{d}(r)
\end{array}\right)
$$

for all $r \vdash n$, where $l_{d}$ is the number of all compositions of $d$ which are Lyndon words over the alphabet $\mathbb{N}$, that is, $l_{d}=\sum_{p \vdash d} h_{p}=\operatorname{dim}_{K} L \cap D_{d}$.

PROOF. As all irreducible $\Delta_{n}$-modules are one-dimensional, we have $\sum_{r \vdash n} c_{r, q}=$ $\operatorname{dim}_{K} \Lambda^{q}$ for all $q \models n$, and also $\sum_{q \vdash n} c_{r, q}=\operatorname{dim}_{K} \mathrm{P}^{r}$ for all $r \vdash n$, by Corollary 2.2 (a). Since $\left\{\omega_{\dot{q}} \mid \dot{q} \approx q\right\}$ is a $K$-basis of $\Lambda^{q}([4,1.3])$, the second equation in (a) follows. It remains to show the second equation in (b). We have $\mathrm{P}^{r}=v_{r} \Delta_{n}=\bigoplus_{q \vdash n} v_{r} \Lambda^{q}$, hence 
$\operatorname{dim} \mathrm{P}^{r}=\sum_{q \vdash n} \operatorname{dim}_{K} v_{r} \Lambda^{q}=\sum_{q \vdash n}\left|\mathfrak{B}_{q}^{r}\right|=\left|\mathfrak{B}^{r}\right|$ for every Poincaré-Birkhoff-Witt basis $\mathfrak{B}$, by (27). We now choose the same basis $\mathfrak{B}$ as in the proof of Corollary 2.1 (a). Then there exists a bijection of $\mathfrak{B}^{r}$ onto the set $\{(w[1], \ldots, w[l]) \mid w[i] \in B, w[i] \models$ $\left.r_{i}, w[1] \geq \cdots \geq w[l]\right\}$, where $l:=|r|$. An application of (1) yields the claim.

Proof of TheOrem 2.2. The equivalence of (i) and (ii) follows from (16) and Corollary 2.1 (b). To prove the equivalence with (iii), we show first:

$$
\text { (iii) implies that } j=|q|-|r| \text {. }
$$

If (iii) holds, then $0 \neq \mathrm{P}^{r} \omega_{q}=\omega_{r} \Lambda^{q}$, hence $|q| \geq|r|$. We put $h:=|q|-|r|$ and obtain $\operatorname{Rad}^{(h+1)} \mathrm{P}^{r} \cap \mathrm{P}^{r} \omega_{q} \subseteq \operatorname{Rad}^{(h+1)} \Delta_{n} \cap \omega_{r} \Lambda^{q}=0, \mathrm{P}^{r} \omega_{q} \subseteq \operatorname{Rad}^{(h)} \Lambda^{q} \cap \mathrm{P}^{r} \subseteq \operatorname{Rad}^{(h)} \mathrm{P}^{r}$, by Proposition 2.3 (b). Therefore neither $\operatorname{Rad}^{(h+1)} \mathrm{P}^{r}$ nor $\mathrm{P}^{r} / \operatorname{Rad}^{(h)} \mathrm{P}^{r}$ has a composition factor isomorphic to $M^{q}$. Hence $h=j$, proving (29). Now the equivalence of (iii) and (ii) follows from (29) and Corollary $2.2(\mathrm{~b})$.

\section{References}

[1] M. D. Atkinson, 'Solomon's descent algebra revisited', Bull. London Math. Soc. 24 (1992), 545551.

[2] Y. A. Bahturin, Identical relations in Lie algebras (VNU Science Press, Utrecht, 1987).

[3] D. Blessenohl and H. Laue, 'Algebraic combinatorics related to the free Lie algebra', in: Actes $29^{e}$ Séminaire Lotharingien (Publ. I. R. M. A., Strasbourg, 1993) pp. 1-21.

[4] — ' 'On the descending Loewy series of Solomon's descent algebra', J. Algebra 180 (1996), 698-724.

[5] L. Dornhoff, Group representation theory, part B (Marcel Dekker, New York, 1972).

[6] A. M. Garsia and C. Reutenauer, 'A decomposition of Solomon's descent algebra', Adv. Math. 77 (1989), 189-262.

[7] I. M. Gelfand, D. Krob, A. Lascoux, B. Leclerc, V. S. Retakh and J.-Y. Thibon, 'Noncommutative symmetric functions', Adv. Math. 112 (1995), 218-348.

[8] S. A. Jennings, 'Central chains of ideals in an associative ring', Duke Math. J. 9 (1942), 341-355.

[9] A. Kerber, Algebraic combinatorics via finite group actions (BI Wissenschaftsverlag, MannheimWien-Zürich, 1991).

[10] M. Lothaire, Combinatorics on words (Addison-Wesley, Reading, 1983).

[11] C. Reutenauer, Free Lie algebras (Oxford Univ. Press, London, 1993).

[12] L. Solomon, 'A Mackey formula in the group ring of a Coxeter group', J. Algebra 41 (1976), 255-268.

[13] E. Witt, 'Treue Darstellung Liescher Ringe', J. Reine Angew. Math. 177 (1937), 152-160.

Mathematisches Seminar der Universität

Ludewig-Meyn-Str. 4

D-24098 Kiel

Germany

e-mail: blessenohl@math.uni-kiel.de,laue@math.uni-kiel.de 
